\title{
Direct Mapping of Krebs Cycle Activity in the Male and Female Brain at Subcellular Resolution Using Soft X-ray Fluorescence
}

\author{
C. Poitry-Yamate, ${ }^{1, *}$, M. Abyaneh ${ }^{2}$, T. Araki ${ }^{2}$, M. Lepore ${ }^{1}$, B. Kaulich ${ }^{2}$ and R. Gruetter ${ }^{1}$ \\ 1. Centre d'Imagerie Biomédicale, Ecole Polytechnique Fédérale de Lausanne, Lausanne, Switzerland \\ 2. Diamond Light Source Ltd, Harwell Science and Innovation Campus, Oxfordshire, UK \\ * Corresponding author, carole.poitry-yamate@epfl.ch
}

Introduction Fluoroacetate (FA), a plant toxin and intermediate product of anticancer agents [1], integrates itself into the Krebs cycle by substituting for acetate to form fluorocitrate. The function of this key metabolic cycle in glucose metabolism is disabled when fluorocitrate binds to aconitase. FA purportedly disrupts the Krebs cycle selectively in non-neuronal cells, i.e. glia/astrocytes, making it the substance of choice by which to assess the importance of glia for brain function in the living animal [2]. However, since acetate, considered a marker of glial oxidative metabolism, can also be transported into neurons [3] begs the question whether FA uptake, metabolism, and compartmentation is glial-selective in vivo, and whether this can be experimentally imaged; as important, is whether the first phase of brain glucose metabolism, i.e. glycolysis is affected. Dynamic cerebral ${ }^{18}$ FDG (glucose) PET in the living rat, and low energy x-ray fluorescence chemical imaging of such brains, albeit freeze substitution-fixed and epon infiltrated, were implemented to address these questions, and enabled testing our working hypothesis that sexual dimorphism of brain structure and function [4] extends to metabolism as well.

Motivation In control animals, the brains of female rats had a significantly greater capacity to phosphorylate ${ }^{18}$ FDG (glucose) than the brains of male rats (Fig. 1d). Moreover, the female brain was particularly impacted by FA at the first step of glycolysis, i.e. phosphorylation of glucose $\left({ }^{18}\right.$ FDG). These dramatic differences between females $(\mathrm{F})$ and males $(\mathrm{M})$ following co-administration of ${ }^{18} \mathrm{FDG}$ and FA (or vehicle solution) intravenously in healthy rats ( $\mathrm{n}>10$ rats per sex) could not be attributed to differences in: FA dose $\left(20 \mathrm{mg} / \mathrm{kg}\right.$ body weight, that targets only glia [2]); the amount of ${ }^{18} \mathrm{FDG}$ reaching the systemic blood (F, 0.30 MBq/g; M, 0.29 MBq/g); initial glycemia (F, $7.39 \pm 0.12 \mathrm{mM}$; M, 7.55 $\pm 0.06 \mathrm{mM}$ ); or to tail vein needle insertion for substance delivery. Might the sexual dimorphic nature of this FA-dependent modulation of brain glycolysis be attributed to neurons? And, to a decrease of carbon flux (so increasing $\mathrm{O}$ and $\mathrm{C}$ content) through the Krebs cycle, thereby decreasing Krebs cycle activity?

Method The aforementioned questions were addressed by performing 2-dimensional distribution mapping of respectively, F, O and $\mathrm{C}$ at $300 \mathrm{~nm}^{2}$ spatial resolution from $2 \mu \mathrm{m}$ thick brain slices. Mapping $\mathrm{C}$ and $\mathrm{N}$ placed the metabolic maps within a cell-type specific context. Measurements under ambient temperature were performed at $830 \mathrm{eV}$, in top-up mode, implementing a $150 \mu \mathrm{m}$ OSA and 500/30 $\mathrm{nm} \mathrm{ZP}$. Samples were raster scanned at $300 \mathrm{~nm}$ steps using dwell times from 2 s to 10 s per pixel.

Main Results Following deconvolution of the fluorescence spectrum per pixel using the PyMCA Hypermet algorithm, color maps were placed in grey, minimizing differences in human color perception, maximizing metabolic information content, and allowing structural differentiation of nuclear regions of the cell from dendritic/endfeet contacts onto the cell soma. In representative control brains (Fig. 1a, top row), the cell soma of pyramidal neurons (outline in TMS) and neighboring astrocyte (C map) were discernible, as were dendritic-axonal processes and extensive bush-like branching of glial processes in the neuropil (*). Cytoplasmic compartments had both a higher $\mathrm{N}$ and $\mathrm{O}$ content, distinguishing it from 
the nuclear compartment (dashed lines). In the female brain (Fig. 1b, middle row) FA uptake was found in the astrocyte processes (region A, TMS image), cell body (outlined, second C map) and endfeet (arrows, first $\mathrm{C}$ map), as well as the cytoplasm of 2 neighboring neurons (outlined, first $\mathrm{C}$ map); oxygen content was largely concentrated in the neuronal cytoplasmic compartment. In the male brain (Fig. 1c, bottom row) FA uptake was localized predominantly to neurons (soma cytoplasm, $\mathrm{N}$ map) and dendrites apposing a neuron cell body (arrows, $\mathrm{C}+\mathrm{N}$ map), where oxygen content was concentrated. In contrast, FA uptake and oxygen content were weakly discernible in the astrocytic processes (outlined, $\mathrm{C}$ map).

\section{a.}

b.
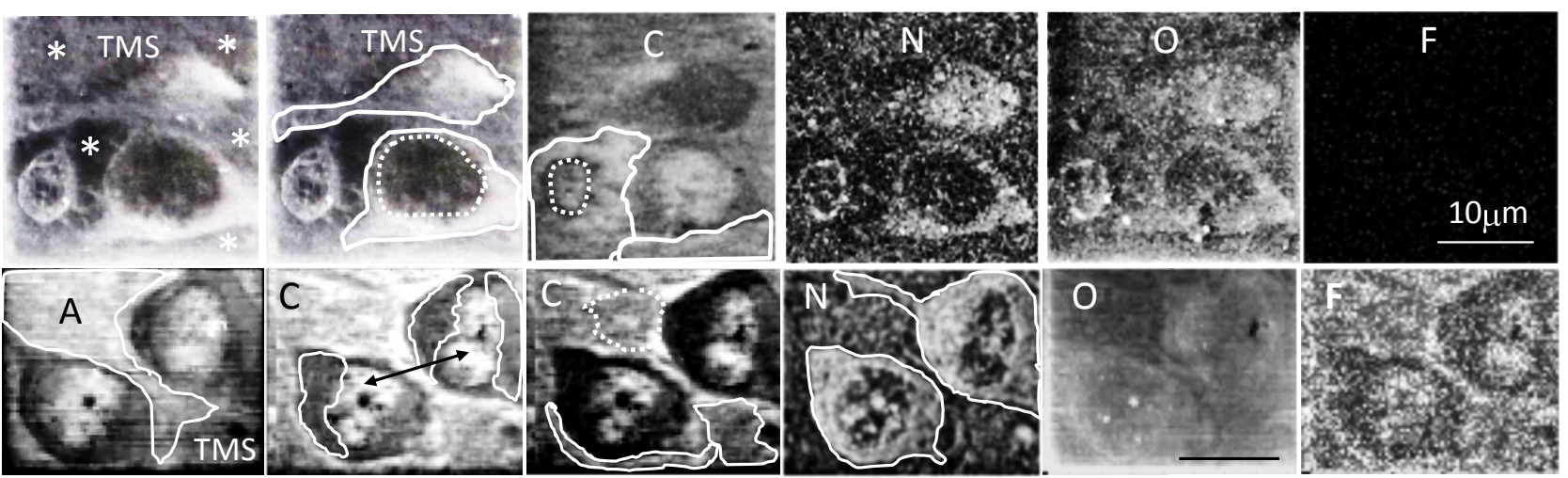

C.
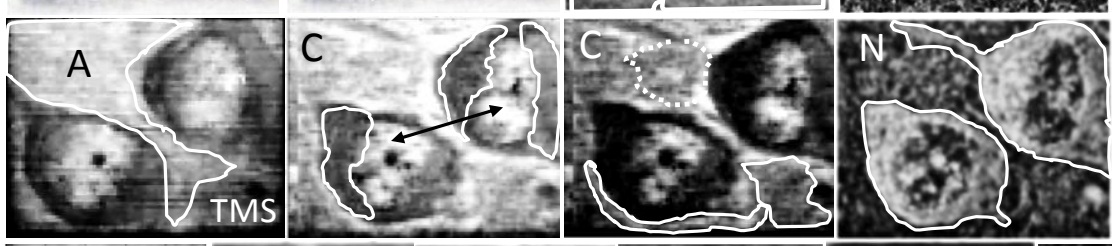

0


Figure 1. X-ray fluorescence chemical mapping of Krebs cycle activity in brain, after dynamic cerebral 18FDG PET imaging, with and without FA. (a) control brain. C/N/O content (average photon counts \pm SEM, n=7maps) was comparable between F and M control brains: C: $6611 \pm 739$; N: $450 \pm 76$; O: 7949 \pm 709. Trend: $\mathrm{O} \gg$ C. (b) female experimental brain. (c) male experimental brain. FA increased the $\mathrm{C} / \mathrm{N} / \mathrm{O} / \mathrm{F}$ content in maps from both female $(\mathrm{n}=8)$ and male $(\mathrm{n}=11)$ brains. Trend: $\mathrm{C} \approx \mathrm{O}$. Female, $\mathrm{C}$ : $45188 \pm 4987 ; \mathrm{N}: 2239 \pm 263 ; \mathrm{O}: 44830 \pm 4521 ; \mathrm{F}: 898 \pm 408$. Male, C: $30068 \pm 3702 ; \mathrm{N}: 1755 \pm 190$; O: $30505 \pm 4387$; F: $2035 \pm 395$. (d) FA's effect on the kinetics of cerebral 18FDG uptake and metabolism between females and males (open symbols), relative to age-matched control brains (filled symbols). FA exerted a notable effect in females by decreasing cerebral glucose phosphorylation. Scale bar: 10 microns. TMS, transmission scale image. Chemical elements are indicated at top map center.

Conclusion The decrease in Krebs cycle activity in both astrocytes and neurons, and the corresponding decrease of brain glucose phosphorylation in the female brain, are in accordance with FA's inhibitory action in a biochemical cascade involving citrate accumulation, phosphofructose kinase inhibition, blockade of glucose phosphorylation, and hyperglycemia [5]. This scenario may be exclusive to astrocytes in the male brain, where cerebral glucose phosphorylation was unaffected, and is compatible with astrocytes being exquisitely sensitive to FA, while the Krebs cycle is unaffected in neurons as they convert FA to fluorocitrate presumably slowly as a means of detoxification. The sexual dimorphism of brain energy metabolism, from the macroscopic to the microscopic length scales, shown here, is a consideration in sex-based approaches to understanding multi-organ metabolic diseases such as diabetes. 


\section{References}

[1] Goncharov et al. (2006) J. Appl. Toxicol. 26:148-161.

[2] Hassel et al. (1997) J Cereb Blood Flow Metab. 17:1230-1238.

[3] Rae et al. (2012) Neurochem Res 37:2541-2553.

[4] McCarthy et al. (2012) J Neurosci. 32:2241-2247.

[5] Bowman (1964) Biochem J. 93:13c-15c.

[6] The authors gratefully acknowledge funding by Diamond Light Source and CIBM-PET core, provision of beam time and reviewer's suggestions. 
https://doi.org/10.1017/S1431927618014010 Published online by Cambridge University Press 